\title{
Selective Changes of Resting-State Brain Oscillations in aMCI: An fMRI Study Using ALFF
}

\author{
Zhilian Zhao, ${ }^{1,2}$ Jie Lu, ${ }^{1,2}$ Xiuqin Jia, ${ }^{1,2}$ Wang Chao, ${ }^{1,2}$ Ying Han, \\ Jianping Jia, ${ }^{3}$ and Kuncheng $\mathrm{Li}^{1,2}$ \\ ${ }^{1}$ Department of Radiology, Xuanwu Hospital of Capital Medical University, Beijing 100053, China \\ ${ }^{2}$ Beijing Key Laboratory of Magnetic Resonance Imaging and Brain Informatics, Beijing 100053, China \\ ${ }^{3}$ Department of Neurology, Xuanwu Hospital of Capital Medical University, Beijing 100053, China
}

Correspondence should be addressed to Kuncheng Li; kuncheng.li@gmail.com

Received 28 January 2014; Accepted 11 March 2014; Published 14 April 2014

Academic Editor: Lijun Bai

Copyright (C) 2014 Zhilian Zhao et al. This is an open access article distributed under the Creative Commons Attribution License, which permits unrestricted use, distribution, and reproduction in any medium, provided the original work is properly cited.

\begin{abstract}
Mild cognitive impairment (MCI) refers to a transitional state between normal aging and dementia and is a syndrome with cognitive decline greater than expected for an individual's age and educational level. As a subtype of MCI, amnestic mild cognitive impairment (aMCI) most often leads to Alzheimer's disease. This study aims to elucidate the altered brain activation in patients with aMCI using resting-state functional magnetic resonance. We observed Frequency-dependent changes in the amplitude of low-frequency fluctuations in aMCI patients $(n=20)$, and normal subjects $(n=18)$. At the same time, we took gray matter volume as a covariate. We found that aMCI patients had decreased amplitude of low-frequency fluctuation signal in left superior temporal gyrus, right middle temporal gyrus, right inferior parietal lobe, and right postcentral gyrus compared to the control group. Specially, aMCI patients showed increased signal in left superior and middle frontal gyrus. Our results suggested that increased activation in frontal lobe of aMCI patients may indicate effective recruitment of compensatory brain resources. This finding and interpretation may lead to the better understanding of cognitive changes of aMCI.
\end{abstract}

\section{Introduction}

The concept of mild cognitive impairment (MCI) refers to subjects who experience cognitive impairments but who are not demented [1]. MCI is a syndrome with cognitive decline greater than expected for an individual's age and educational level but not interfering notably with activities of daily living. The prevalence of MCI is about $15 \%$ in adults older than 65 years and more than half of MCI patients progress to dementia within 5 years. The common outcome of nonamnestic MCI is frontotemporal dementia or dementia with Lewy bodies. Patients with the amnestic subtype of MCI (aMCI) have an annual conversion rate of $6-25 \%$ to Alzheimer's disease (AD). As such, aMCI has been regarded as a prodromal stage of $\mathrm{AD}$ [2-6]. Over the past decade, significant progress has been accomplished in our understanding of its epidemiology, risk factors, natural history, and treatment. Although there remain some controversies surrounding $\mathrm{MCI}$, it is increasingly recognized that $\mathrm{MCI}$ should be handled as a clinically defined condition. Because the standard diagnostic procedure of aMCI primarily relies on neuropsychological examinations, there is strong demand to develop neuroimaging techniques as reliable surrogate MCI markers. Whereas structural MRI provides important diagnostic and prognostic information, fMRI remains promising as an imaging marker of MCI, including aMCI.

Recently, low-frequency fluctuations (LFF) fMRI has gained increased attention based on observations using fMRI approaches and direct current coupled electroencephalographic scalp recordings [7-9]. Spatially organized and temporally coherent fluctuations in the low-frequency range $(0.01-0.1 \mathrm{~Hz})$ have been at the center of attention, as the BOLD signal displays a spatial structure similar to task function-related activation [10-12].

Most studies of resting-state functional magnetic resonance imaging (fMRI) have applied the temporal correlation 
in the time courses to study the functional connectivity between different brain regions. Biswal and coworkers have shown that spontaneous low-frequency $(<0.08 \mathrm{~Hz})$ fluctuation (LFF) is highly synchronous among motor cortices [10]. Recently, resting-state synchronization has also been investigated in patients [13-16] and in healthy subjects [1719]. The power of (LFF) may also be used as a biomarker to assess cerebral spontaneous activity [20]. ALFF is defined as the total power within the frequency range between 0.01 and $0.1 \mathrm{~Hz}$. Our study aims to evaluate the ALFF signal in reflecting cerebral physiological states in aMCI patients and healthy subjects. We evaluate whether the ALFF abnormalities in aMCI have similar distribution pattern as independent component analysis (ICA) approach [21]. Several studies had shown grey matter loss in aMCI or MCI [22-25] and regional brain atrophy may lead to artificial reduction in low-frequency fluctuation [26]. In order to directly test our hypothesis and improve the statistical strength, we took gray matter volume as a covariate.

\section{Materials and Methods}

2.1. Study Population. Thirty-eight right-handed subjects were recruited. Participants were divided into two groups based on their clinical profiles: twenty participants were classified as aMCI patients and the other eighteen as healthy controls. Patients were recruited from a memory clinic at the Department of Neurology and healthy controls were recruited from a community investigation of epidemiological research. Informed consent was approved by the Medical Research Ethics Committee of Xuanwu Hospital and obtained from all subjects. Prior to resting-state fMRI scanning, examination of each subject included medical history, neurological examination, informant interview, neuropsychological assessment 4 including mini-mental state examination (MMSE), clinical dementia rating (CDR), activity of daily living scale, Hachinski ischemic scale, Hamilton rating scale for depression, auditory verbal learning test (AVLT), structural MRI, and standard laboratory tests.

aMCI diagnosis was established according to the criteria for amnestic MCI $[5,6]$. To be diagnosed as having MCI, patients had to fulfill the following criteria: (a) impaired memory performance on a normalized objective verbal memory test, (b) recent history of symptomatic worsening in memory, (c) normal or near-normal performance on global cognitive tests [mini-mental state examination (MMSE) score > 24] as well as on activities of daily living scale, (d) global rating of 0.5 on the CDR scale, with a score of at least 0.5 on the memory domain, and (e) absence of dementia. Stroke, psychiatric diseases, drug abuse, moderate to serious hypertension, and systematic diseases were ruled out. Memory complaints or neurological deficiencies were not observed in the healthy controls with normal conventional brain MR imaging and an MMSE score $\geq 28$. Demographics and neuropsychological findings of aMCI patients and healthy elderly are shown in Table 1. Demographics of aMCI patients and healthy controls, including age, sex, and education years, were matched between the two groups.
TABLE 1: Demographics and neuropsychological findings of aMCI patients and healthy elderly.

\begin{tabular}{lcc}
\hline & $\begin{array}{c}\text { aMCI patient } \\
(n=20)\end{array}$ & $\begin{array}{c}\text { Healthy elderly } \\
(n=18)\end{array}$ \\
\hline Age & $65.11 \pm 9.92$ & $66.81 \pm 7.43$ \\
Female/male & $12 / 8$ & $10 / 8$ \\
Education (y) & $11.84 \pm 3.32$ & $12.02 \pm 2.93$ \\
MMSE & $25.21 \pm 2.24$ & $29.31 \pm 1.22$ \\
AVLT, learning A1 & $7.22 \pm 1.73$ & $3.95 \pm 1.82$ \\
AVLT, learning A2 & $9.39 \pm 2.73$ & $5.15 \pm 1.42$ \\
AVLT, learning A3 & $10.94 \pm 2.15$ & $6.05 \pm 2.54$ \\
AVLT, delayed recall A4 & $11.33 \pm 2.52$ & $4.75 \pm 3.51$ \\
AVLT, recognition A5 & $13.06 \pm 1.80$ & $7.80 \pm 3.53$ \\
CDR & 0.5 & 0 \\
\hline
\end{tabular}

The age of participants was equally distributed between the two diagnostic groups $(t=2.06, P=0.57$, two-sample two-tailed $t$-test) with similar medians and ranges. However, the groups were significantly different with regard to MMSE scores and AVLT scores $(P<0.001$, two-sample two-tailed $t$-test).

2.2. Data Acquisition. MRI data were collected on a $3 \mathrm{~T}$ scanner (Siemens, Trio, Erlangen, Germany), with an eightchannel receiver coil. Subjects were instructed to keep their eyes closed and to refrain from initiating goal-directed, attention-demanding activity during the scanning sessions, and resting-state fMRI was acquired. fMRI was acquired using gradient echo planner imaging (EPI) for a $6 \mathrm{~min}$ and $20 \mathrm{~s}$ period, resulting in a total of 124 volumes (repetition time (TR)/echo time $(\mathrm{TE})=3000 / 30 \mathrm{~ms}$, flip angle $=90^{\circ}$, field of view $(\mathrm{FOV})=256 \times 256 \mathrm{~mm}^{2}$, matrix size $=64 \times 64$, 28 slices, slice thickness $=4 \mathrm{~mm}$, and $0 \mathrm{~mm}$ interslice gap). A $\mathrm{T}_{1}$ WI anatomical dataset was obtained from each subject using a magnetization-prepared rapid acquisition gradient echo sequence $(\mathrm{TR} / \mathrm{TE}=1900 / 2.2 \mathrm{~ms}$, inversion time $(\mathrm{TI})=$ $900 \mathrm{~ms}$, flip angle $=9^{\circ}, \mathrm{FOV}=256 \times 256 \mathrm{~mm}^{2}$, matrix size $=$ $224 \times 256,176$ slices, voxel size $=1 \times 1 \times 1 \mathrm{~mm}^{3}$ ). According to the inclusion criteria, $\mathrm{T}_{2} \mathrm{WI}$ and FLAIR scans were reviewed to exclude the presence of remarkable macroscopic brain abnormalities.

2.3. Voxel-Based Morphometry Data Processing. Structural MRI data analysis was performed using an optimized VBM protocol (http://dbm.neuro.uni-jena.de/vbm/) under SPM5 (http://www.fil.ion.ucl.ac.uk/spm/), which included slice timing, motion correction, spatial normalization, and smoothing.

Images were segmented into grey matter, white matter, and cerebral spinal fluid (CSF) [27]. The images were then normalized to the Montreal Neurological Institute (MNI) template, and then the parameters were applied to normalize individual $\mathrm{T}_{1}$ images separately. The fully normalized images were once again segmented into grey matter, white matter, and CSF. The normalized and modulated white matter and 
gray matter images were smoothed using a $4 \mathrm{~mm} \times 4 \mathrm{~mm} \times$ $4 \mathrm{~mm}$ full-width half-maximum (FWHM) Gaussian kernel for subsequent statistical analysis. Then, the grey matter volume of the aMCI patients and the normal controls was calculated. The result was also entered into the following statistical analysis to examine the effects of GM atrophy on the functional results.

2.4. Resting-State fMRI Data Processing and Statistics. Functional MRI data were processed using the statistical parametric mapping (SPM5, http://www.fil.ion.ucl.ac.uk/ $\mathrm{spm} /$ ), which included slice timing, motion correction, and spatial normalization. ALFF analysis was performed by using resting-state fMRI data analysis toolkit (http://restingfmri.sourceforge.net/). The procedure for calculating the ALFF is similar to the previous studies $[20,28]$. The filtered time series was transformed to a frequency domain with a fast Fourier transform (FFT) (parameters: taper percent $=0$, FFT length $=$ shortest) and the power spectrum was then obtained. The square root was thus calculated at each frequency of the power spectrum and the averaged square root was obtained across $0.01-0.08 \mathrm{~Hz}$ at each voxel. This averaged square root was taken as the ALFF. For standardization purposes, the ALFF of each voxel was divided by the global mean ALFF value within a brain mask, which was obtained from the intersection of the brain of all subjects' $\mathrm{T}_{1}$ images. The analysis included the grey matter as a covariate. A two-sample $t$-test was performed to test the ALFF difference between aMCI patients and normal controls. Voxels with a corrected $P$ value $<0.01$ (single voxel threshold of $P<0.05$ and cluster size $540 \mathrm{~mm}^{3}$, using the AlphaSim program with parameters: FWHM $=8 \mathrm{~mm}$, with mask) were considered significant.

\section{Results}

3.1. Demography and Neuropsychological Test. Demographic characteristics and neuropsychological scores were shown in Table 1. There were no significant differences between the two groups in gender, age, and years of education, but the MMSE and AVLT (auditory verbal learning test) scores were significantly different $(P<0.05)$ between the two groups.

\subsection{Brain Regions of Decreased Gray Matter Volume between} the Two Groups. To assess possible causes of reduced functional activity, we analyzed our data for structural differences between both study groups. The aMCI patients showed widespread reduction in gray matter volume in the right uncus, the bilateral inferior, superior, and middle frontal gyrus, the bilateral medial temporal gyrus, the left inferior temporal gyrus, the left superior temporal gyrus, the right superior parietal gyrus, and the left middle occipital gyrus $(P<0.001$, corrected) (Figures 1 and 2, Table 2). Some of these areas did overlap with those regions found to be altered in the patient group using ALFF.

3.3. Whole Brain Functional Alteration Data: The BetweenGroup Differences. Figure 2 shows the statistical map resulting from comparison of the changes of ALFF in different
TABLE 2: Areas of gray matter loss in aMCI patients compared with healthy controls.

\begin{tabular}{cccccccc}
\hline \multirow{2}{*}{ Region } & \multirow{2}{*}{ BA } & \multirow{2}{*}{ Voxels } & \multicolumn{3}{c}{ MNI } & \multirow{2}{*}{-score } \\
& & & $x$ & $y$ & $z$ & \\
\hline Left MTG & 35 & 23 & -18 & -27 & -24 & 5.26 \\
Right MTG & 30 & 14 & 15 & -33 & -12 & 4.05 \\
NC-MCI & Left MOG & 34 & 12 & -9 & -3 & -24 & 3.93 \\
Right MFG & 37 & 12 & 60 & -45 & -9 & 3.79 \\
Right uncus & 44 & 12 & 63 & 6 & 21 & 3.75 \\
Left IFG & 10 & 10 & -30 & 51 & -3 & 3.32 \\
Right SFG & 22 & 14 & 60 & 6 & 3 & 3.26 \\
\hline
\end{tabular}

*MTG: medial temporal gyrus; MOG: middle occipital gyrus; MFG: middle frontal gyrus; IFG: inferior frontal gyrus; SFG: superior frontal gyrus.

brain areas in healthy elderly versus aMCI patients. ALFF was higher in controls than patients in left superior temporal gyrus, right middle temporal gyrus, right inferior parietal lobe, and right postcentral gyrus. ALFF was significantly higher in patients than controls in left superior, middle frontal gyrus (see Figure 2 and Table 3).

\section{Discussion}

In the current study, we reported abnormal ALFF in aMCI patients compared to healthy controls. ALFF was higher in controls than patients in the left superior temporal gyrus, right middle temporal gyrus, right inferior parietal lobe, and right postcentral gyrus. Patients had higher ALFF than controls in left superior, middle frontal gyrus. Thus, our data suggested that there are abnormalities in LFOs in aMCI patients. The current findings add to a literature suggesting abnormalities of neural synchrony in aMCI and extend these findings to the LFO domain.

The reduced LFO in the IPL and the temporal gyrus is consistent with previous studies of aMCI. More recently, functional imaging studies have suggested that memory processes are subserved by a set of distributed, large-scale neural networks. Specific regions of the default network are selectively vulnerable to early amyloid deposition in $\mathrm{AD}$. The lateral parietal and temporoparietal areas are involved in the default network [29]. Using ICA, [21] found that right inferior parietal lobule exhibited decreased functional activity in aMCI compared to normal control, and [30] found reduced activity in the patient group in bilateral superior parietal lobes. Previous studies have demonstrated that some regions constitute a structurally and functionally connected neuronal network that supports the default function of the human brain. The IPL is one of the brain regions that constitute the major posterior extent of the default mode network (DMN). Reduced right IPL activity indicated impaired memory functional system in aMCI patients. Episodic memory function is severely affected in $\mathrm{AD}$ and is also the key early marker for prodromal stages such as MCI [31]. This may suggest that the aMCI is prodromal stage of $\mathrm{AD}$. 
TABLE 3: Resting-state activities in controls and aMCI patients (amplitude of low-frequency fluctuations).

\begin{tabular}{|c|c|c|c|c|c|c|c|}
\hline \multirow{2}{*}{$\begin{array}{l}\text { Condition } \\
\text { NC-MCI }\end{array}$} & \multirow{2}{*}{$\begin{array}{c}\text { Connected regions } \\
\text { Left superior temporal gyrus }\end{array}$} & \multirow{2}{*}{$\begin{array}{l}\mathrm{BA} \\
22\end{array}$} & \multirow{2}{*}{$\begin{array}{c}\text { Cluster } \\
37\end{array}$} & \multirow{2}{*}{$\begin{array}{c}t \text {-score } \\
3.10\end{array}$} & \multicolumn{3}{|c|}{ Coordinates (MNI) } \\
\hline & & & & & -45 & -3 & -3 \\
\hline & & & & 2.69 & -45 & 6 & -6 \\
\hline & Right middle temporal gyrus & 21 & 16 & 2.85 & 60 & -42 & 0 \\
\hline & & & & 2.67 & 63 & -48 & 6 \\
\hline & Right inferior parietal lobe & 40 & 16 & 2.577 & 51 & -42 & 60 \\
\hline & Right postcentral gyrus & 20 & & 2.303 & 51 & -33 & 60 \\
\hline & Right inferior parietal lobe & 40 & & 2.131 & 42 & -45 & 57 \\
\hline \multirow[t]{3}{*}{ MCI-NC } & Left middle frontal gyrus & 10 & 28 & 4.148 & -36 & 57 & -12 \\
\hline & Left superior frontal gyrus & 11 & & 3.577 & -30 & 57 & -3 \\
\hline & & & & 2.43 & -24 & 63 & -3 \\
\hline
\end{tabular}

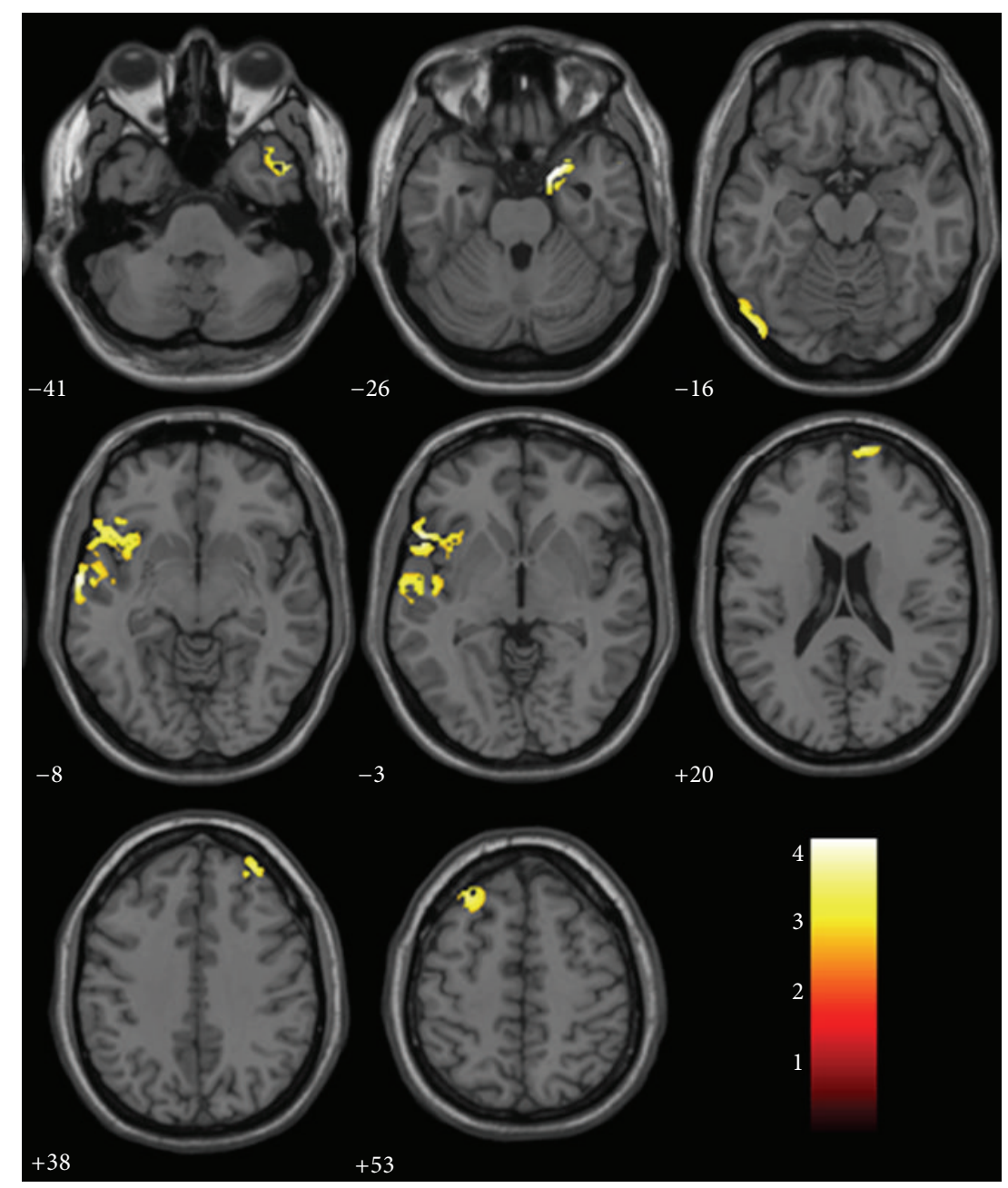

FIGURE 1: VBM analysis showed brain regions of significant reduction of gray matter volume in aMCI patients relative to controls on axial position images. Reader's right is subjects' right.

An interesting finding of the present study is that, in aMCI without motor clinical impairment, LFOs abnormalities occur also in the motor system, mainly in right postcentral gyrus. The abnormal change in motor system may resemble those described for the cognitive network. Recent evidence indicates that part of classical motor areas may have nonmotor cognitive functions in addition to the well-known motor functions [32]. It is well known that the parietal cortex, which has extensive connections with regions of the frontal lobes, where it sends rich sensory information for movement control, is involved in the elaboration of somatosensory inputs and in movement preparation and planning [33]. 


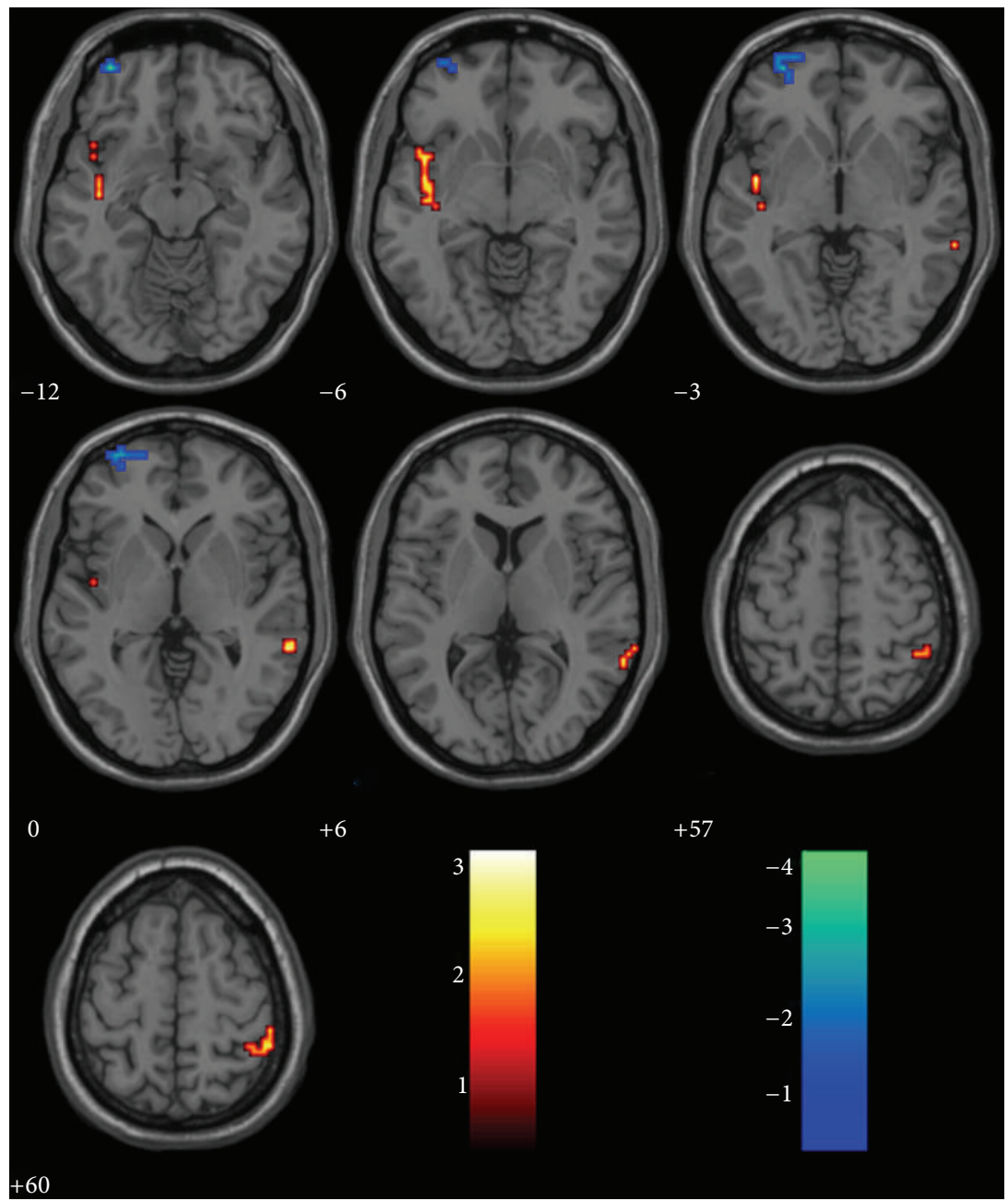

FIGURE 2: Decreased and increased activity in aMCI patients shown in axial projection compared to the healthy elder. Hot: NC-aMCI (decreased); winter: aMCI-NC (increased).

These results may suggest an overactivation of selected areas of the sensorimotor network. However, there may be other explanations that we have not known. More work needs to be done to study the role of the postcentral gyrus in the aMCI patients.

The areas of increased amplitude in patients are mainly located in left frontal regions previously associated with abnormal function in this disorder. The frontal cortices are key regions involved in human memory processing $[21,34]$. This is consistent with the assumption that $\mathrm{AD}$ and $\mathrm{MCI}$ patients may be able to use additional neural resources in prefrontal regions to compensate for losses in cognitive function $[21,35,36]$. Critically, activity in this network of regions was correlated with the ability of the patients to perform the tasks accurately. Patients who had more activity in bilateral prefrontal areas were better able to perform tasks of semantic and episodic memory [35, 37]. However, it still remains unclear whether these alterations may be compensatory to maintain memory performance in the setting of early $A D$ pathology or instead represent evidence of excitotoxicity and impending neuronal failure.
There were several limitations in the current study that need to be addressed. First, the education years among the aMCI subjects we selected were large, between 0 (illiteracy) and 19; this may have confounding effects on the results; in future studies we would group our subjects across their education with more aMCI patients. Second, the subjects were instructed to keep their eyes closed during the resting scan; subjects may have looked at something subconsciously and we did not obtain such behavioral data, or some subjects sleep during the examination. Previous studies have suggested that the alpha power is related to different resting conditions 13 $[38,39]$. Future studies would benefit from the use of eye tracking or visual monitoring equipment during the restingstate session.

\section{Conclusions}

In conclusion, we have demonstrated ALFF differences in aMCI patients using functional MRI method. This restingstate fMRI study suggests that the abnormal spontaneous 
activity of these regions may indicate the underlying pathophysiology of aMCI. Research is ongoing to determine if these early alterations will serve as sensitive predictors of clinical decline and, eventually, as markers of aMCI progress to AD.

\section{Conflict of Interests}

The authors declare that there is no conflict of interests regarding the publication of this paper.

\section{Acknowledgment}

This work was partially supported by the Natural Science Foundation of China, Grant nos. 30930029 and 30970823.

\section{References}

[1] H. H. Feldman and C. Jacova, "Mild cognitive impairment," American Journal of Geriatric Psychiatry, vol. 13, no. 8, pp. 645655, 2005.

[2] D. A. Bennett, R. S. Wilson, J. A. Schneider et al., "Natural history of mild cognitive impairment in older persons," Neurology, vol. 59, no. 2, pp. 198-205, 2002.

[3] K. Blennow, M. J. de Leon, and H. Zetterberg, "Alzheimer's disease," The Lancet, vol. 368, no. 9533, pp. 387-403, 2006.

[4] C. DeCarli, "Mild cognitive impairment: prevalence, prognosis, aetiology, and treatment," The Lancet Neurology, vol. 2, no. 1, pp. 15-21, 2003.

[5] R. C. Petersen, G. E. Smith, S. C. Waring, R. J. Ivnik, E. G. Tangalos, and E. Kokmen, "Mild cognitive impairment: clinical characterization and outcome," Archives of Neurology, vol. 56, no. 3, pp. 303-308, 1999.

[6] R. C. Petersen, R. Doody, A. Kurz et al., "Current concepts in mild cognitive impairment," Archives of Neurology, vol. 58, no. 12, pp. 1985-1992, 2001.

[7] C. Demanuele, C. J. James, and E. J. Sonuga-Barke, "Distinguishing low frequency oscillations within the $1 / \mathrm{f}$ spectral behaviour of electromagnetic brain signals," Behavioral and Brain Functions, vol. 3, article 62, 2007.

[8] M. D. Fox and M. E. Raichle, "Spontaneous fluctuations in brain activity observed with functional magnetic resonance imaging," Nature Reviews Neuroscience, vol. 8, no. 9, pp. 700-711, 2007.

[9] X.-N. Zuo, A. Di Martino, C. Kelly et al., "The oscillating brain: complex and reliable," NeuroImage, vol. 49, no. 2, pp. 1432-1445, 2010.

[10] B. Biswal, F. Z. Yetkin, V. M. Haughton, and J. S. Hyde, "Functional connectivity in the motor cortex of resting human brain using echo-planar MRI," Magnetic Resonance in Medicine, vol. 34, no. 4, pp. 537-541, 1995.

[11] M. D. Fox, A. Z. Snyder, J. L. Vincent, M. Corbetta, D. C. Van Essen, and M. E. Raichle, "The human brain is intrinsically organized into dynamic, anticorrelated functional networks," Proceedings of the National Academy of Sciences of the United States of America, vol. 102, no. 27, pp. 9673-9678, 2005.

[12] V. Kiviniemi, T. Starck, J. Remes et al., "Functional segmentation of the brain cortex using high model order group PICA," Human Brain Mapping, vol. 30, no. 12, pp. 3865-3886, 2009.

[13] S.-J. Li, Z. Li, G. Wu, M.-J. Zhang, M. Franczak, and P. G. Antuono, "Alzheimer disease: evaluation of a functional MR imaging index as a marker," Radiology, vol. 225, no. 1, pp. 253$259,2002$.
[14] M. D. Greicius, G. Srivastava, A. L. Reiss, and V. Menon, "Default-mode network activity distinguishes Alzheimer's disease from healthy aging: evidence from functional MRI," Proceedings of the National Academy of Sciences of the United States of America, vol. 101, no. 13, pp. 4637-4642, 2004.

[15] Z. Wang, C. Yan, C. Zhao et al., "Spatial patterns of intrinsic brain activity in mild cognitive impairment and alzheimer's disease: a resting-state functional MRI study," Human Brain Mapping, vol. 32, no. 10, pp. 1720-1740, 2011.

[16] Y. Han, J. Wang, Z. Zhao et al., "Frequency-dependent changes in the amplitude of low-frequency fluctuations in amnestic mild cognitive impairment: a resting-state fMRI study," NeuroImage, vol. 55, no. 1, pp. 287-295, 2011.

[17] D. Cordes, V. M. Haughton, K. Arfanakis et al., "Frequencies contributing to functional connectivity in the cerebral cortex in "resting-state" data," American Journal of Neuroradiology, vol. 22, no. 7, pp. 1326-1333, 2001.

[18] M. J. Lowe, B. J. Mock, and J. A. Sorenson, "Functional connectivity in single and multislice echoplanar imaging using restingstate fluctuations," NeuroImage, vol. 7, no. 2, pp. 119-132, 1998.

[19] V. Kiviniemi, J.-H. Kantola, J. Jauhiainen, and O. Tervonen, "Comparison of methods for detecting nondeterministic BOLD fluctuation in fMRI," Magnetic Resonance Imaging, vol. 22, no. 2, pp. 197-203, 2004.

[20] H. Yang, X.-Y. Long, Y. Yang et al., "Amplitude of low frequency fluctuation within visual areas revealed by resting-state functional MRI," NeuroImage, vol. 36, no. 1, pp. 144-152, 2007.

[21] Z. Qi, X. Wu, Z. Wang et al., "Impairment and compensation coexist in amnestic MCI default mode network," NeuroImage, vol. 50, no. 1, pp. 48-55, 2010.

[22] M. Bozzali, M. Filippi, G. Magnani et al., "The contribution of voxel-based morphometry in staging patients with mild cognitive impairment," Neurology, vol. 67, no. 3, pp. 453-460, 2006.

[23] G. Chételat, B. Landeau, F. Eustache et al., "Using voxelbased morphometry to map the structural changes associated with rapid conversion in MCI: a longitudinal MRI study," NeuroImage, vol. 27, no. 4, pp. 934-946, 2005.

[24] A. Hämäläinen, S. Tervo, M. Grau-Olivares et al., "Voxelbased morphometry to detect brain atrophy in progressive mild cognitive impairment," NeuroImage, vol. 37, no. 4, pp. 1122-1131, 2007.

[25] L. Rami, B. Gómez-Anson, G. C. Monte, B. Bosch, R. SánchezValle, and J. L. Molinuevo, "Voxel based morphometry features and follow-up of amnestic patients at high risk for Alzheimer's disease conversion," International Journal of Geriatric Psychiatry, vol. 24, no. 8, pp. 875-884, 2009.

[26] Y. He, L. Wang, Y. F. Zang et al., "Regional coherence changes in the early stages of Alzheimer's disease: a combined structural and resting-state functional MRI study," NeuroImage, vol. 35, no. 2, pp. 488-500, 2007.

[27] J. Talairach and P. Tournoux, Co-Planar Stereotaxic Atlas of the Human Brain, Thieme Medical, New York, NY, USA, 1987.

[28] Y. F. Zang, Y. He, C. Z. Zhu et al., "Altered baseline brain activity in children with ADHD revealed by resting-state functional MRI," Brain \& Development, vol. 29, no. 2, pp. 83-91, 2007.

[29] R. L. Buckner, "Memory and executive function in aging and ad: Multiple factors that cause decline and reserve factors that compensate," Neuron, vol. 44, no. 1, pp. 195-208, 2004.

[30] C. Sorg, V. Riedl, M. Muhlau et al., "Selective changes of restingstate networks in individuals at risk for Alzheimer's disease," Proceedings of the National Academy of Sciences of the United States of America, vol. 104, no. 47, pp. 18760-18765, 2007. 
[31] L. A. Rabin, N. Paré, A. J. Saykin et al., "Differential memory test sensitivity for diagnosing amnestic mild cognitive impairment and predicting conversion to Alzheimer's disease," Neuropsychology, Development, and Cognition. B, Aging, Neuropsychology and Cognition, vol. 16, no. 3, pp. 357-376, 2009.

[32] T. Hanakawa, M. Honda, N. Sawamoto et al., "The role of rostral Brodmann area 6 in mental-operation tasks: an integrative neuroimaging approach," Cerebral Cortex, vol. 12, no. 11, pp. 11571170, 2002.

[33] G. Rizzolatti, L. Fogassi, and V. Gallese, "Parietal cortex: from sight to action," Current Opinion in Neurobiology, vol. 7, no. 4, pp. 562-567, 1997.

[34] R. L. Buckner, J. R. Andrews-Hanna, and D. L. Schacter, "The brain's default network: anatomy, function, and relevance to disease," Annals of the New York Academy of Sciences, vol. 1124, pp. 1-38, 2008.

[35] C. L. Grady, A. R. McIntosh, S. Beig, M. L. Keightley, H. Burian, and S. E. Black, "Evidence from functional neuroimaging of a compensatory prefrontal network in Alzheimer's disease," The Journal of Neuroscience, vol. 23, no. 3, pp. 986-993, 2003.

[36] L. Wang, Y. Zang, Y. He et al., "Changes in hippocampal connectivity in the early stages of Alzheimer's disease: evidence from resting state fMRI," NeuroImage, vol. 31, no. 2, pp. 496504, 2006.

[37] A. L. Bokde, M. Karmann, C. Born et al., "Altered brain activation during a verbal working memory task in subjects with amnestic mild cognitive impairment," Journal of Alzheimer's Disease, vol. 21, no. 1, pp. 103-118, 2010.

[38] B. Feige, K. Scheffler, F. Esposito, F. Di Salle, J. Hennig, and E. Seifritz, "Cortical and subcortical correlates of electroencephalographic alpha rhythm modulation," Journal of Neurophysiology, vol. 93, no. 5, pp. 2864-2872, 2005.

[39] Q. Zou, X. Long, X. Zuo et al., "Functional connectivity between the thalamus and visual cortex under eyes closed and eyes open conditions: a resting-state fMRI study," Human Brain Mapping, vol. 30, no. 9, pp. 3066-3078, 2009. 


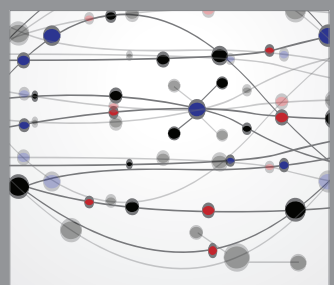

The Scientific World Journal
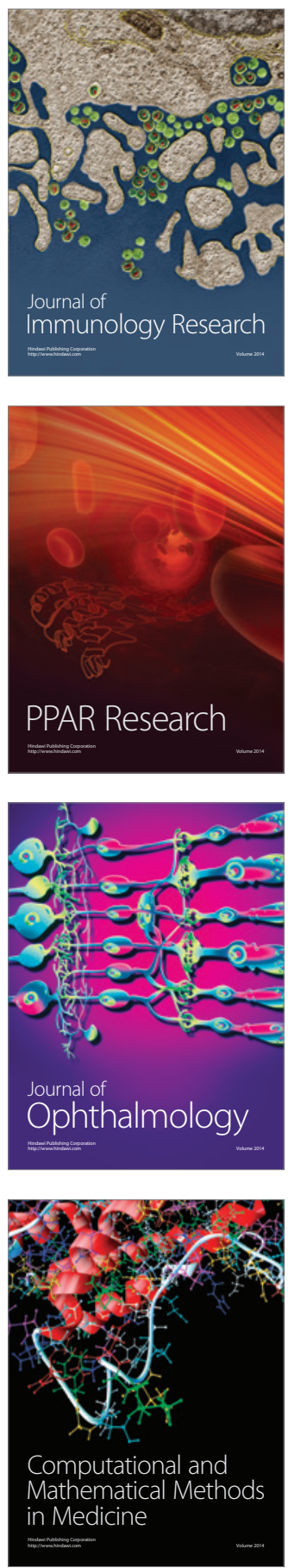

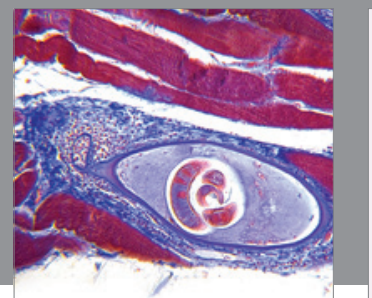

Gastroenterology

Research and Practice
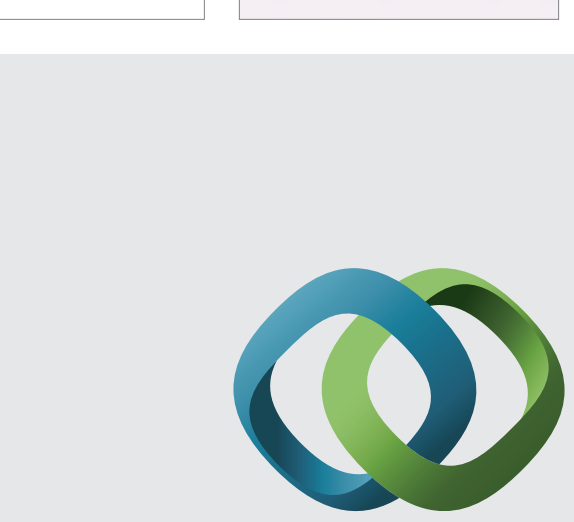

\section{Hindawi}

Submit your manuscripts at

http://www.hindawi.com
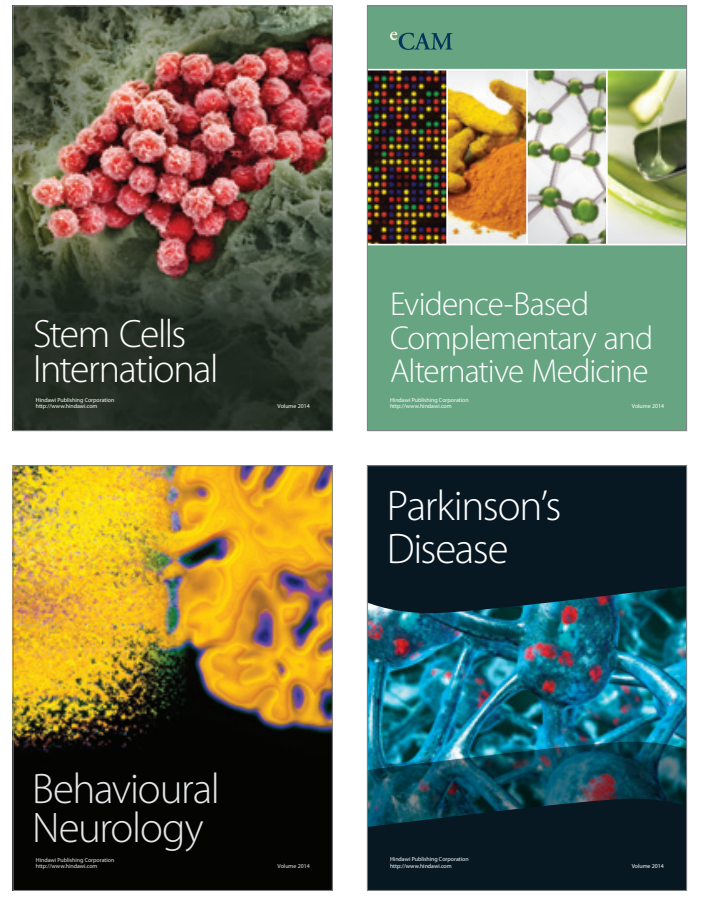
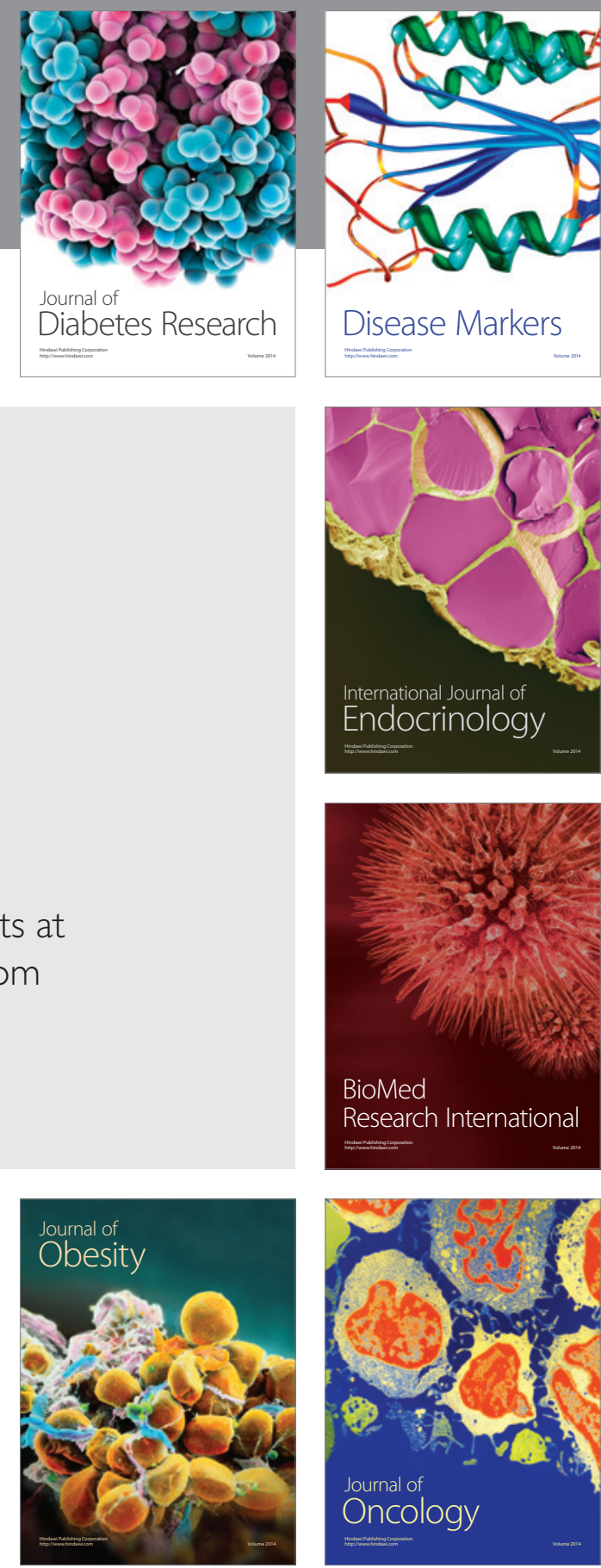

Disease Markers
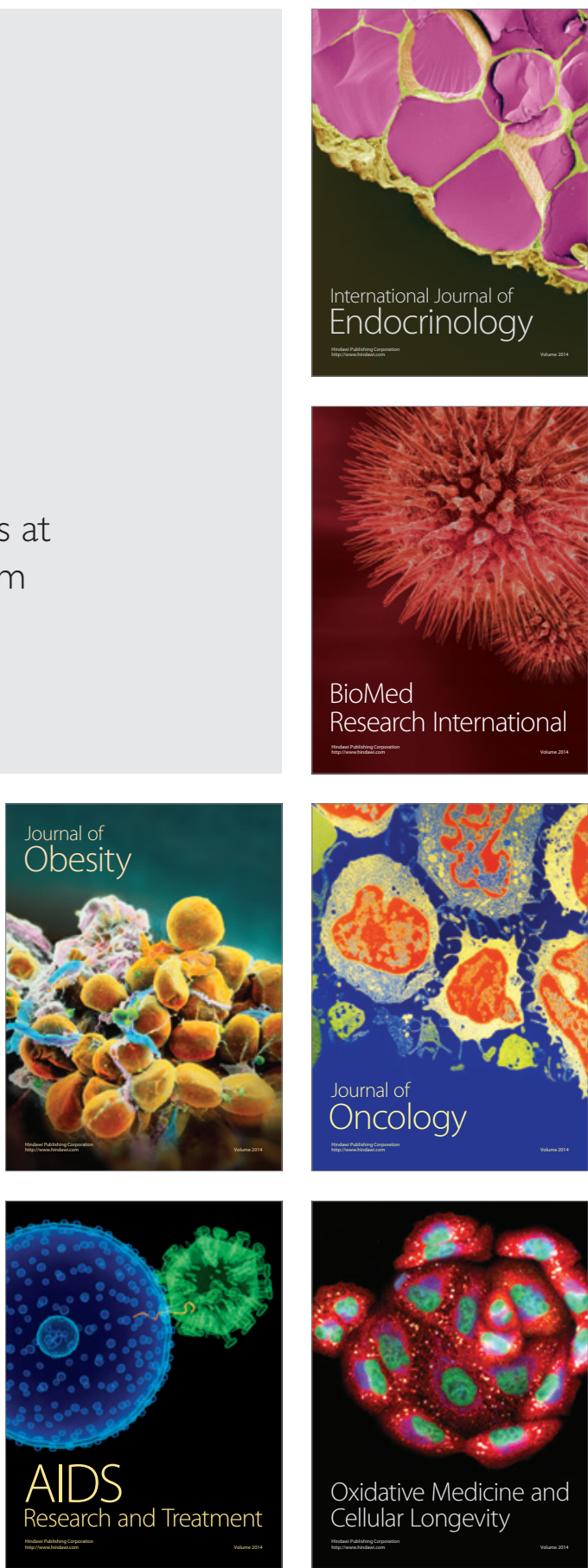